\title{
Rationale and budget impact of bimonthly use of Cetuximab in patients with recurrent and/or metastatic head and neck cancer
}

\author{
FS Mennini ${ }^{1,2} \mid$ A Marcellusi $\mathrm{PhD}^{1,2} \mid$ G Fabiano MRes ${ }^{1,2} \mid$ F Platini $\mathrm{MD}^{3} \mid \mathrm{P}^{3}$ Bossi $\mathrm{MD}^{3}$ (6)
}

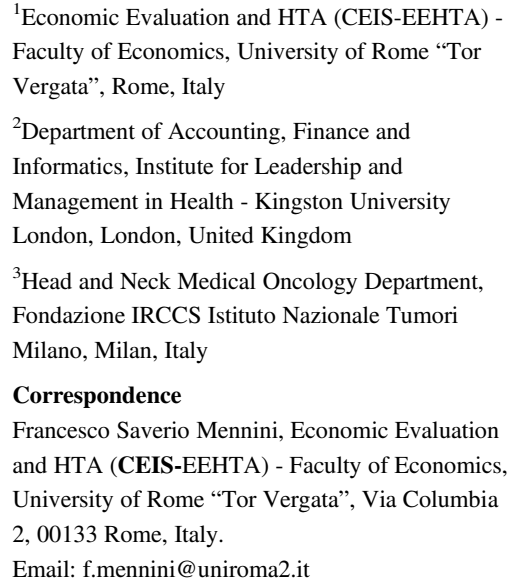

\begin{abstract}
Background: In recurrent and/or metastatic head and neck squamous cell cancer, Cetuximab is administered once a week, followed by weekly doses. We present the clinical rationale of a different schedule of maintenance Cetuximab and we estimate the potential economic benefits on the health care budget from a societal perspective in Italy.

Methods: A budget impact (BI) excel-based model was developed comparing a base case scenario of $100 \%$ weekly administration with a dose of $250 \mathrm{mg} / \mathrm{m}^{2}$ to an everyother-week (EOW) administration at $50 \%$ or $100 \%$ with a dose of $500 \mathrm{mg} / \mathrm{m}^{2}$.

Results: In the EOW, 50\% scenario it was calculated a cost reduction of $€ 347000$ of which $70 \%$ attributable to indirect costs, increasing to $€ 694000$ after 4 months. Conclusions: In our analysis, we showed that this simplified schedule could also reduce the costs of treatments both for the health system (direct costs) and for the society (indirect costs).
\end{abstract}

\section{KEYWORDS}

budget, cetuximab, economic benefits, every-other week, head and neck cancer

\section{1 | INTRODUCTION}

Cetuximab is a chimeric monoclonal immunoglobulin $\mathrm{G}_{1}$ antibody inhibitor of the epidermal growth factor receptor. Because of the substantial benefit in progression-free survival (PFS) and overall survival (OS), ${ }^{1,2}$ Cetuximab is indicated for the treatment of head and neck squamous cell cancer (HNSCC) both in combination with radiotherapy for locally advanced disease and with platinum-based chemotherapy for recurrent and/or metastatic (RM) disease. In all indications, Cetuximab is administered by intravenous infusion once a week at an initial dose of $400 \mathrm{mg} / \mathrm{m}^{2}$, followed by weekly doses of $250 \mathrm{mg} / \mathrm{m}^{2.3}$ Although the weekly schedule was validated in clinical studies, the long Cetuximab half-life of 66-98 hours makes its administration every other week (EOW) theoretically possible.

Pharmacodynamic (PD) and pharmacokinetic (PK) studies have demonstrated the bioequivalence as well as the efficacy of a EOW dosing schedule, when Cetuximab was used as a treatment of colorectal cancer. ${ }^{4-7}$ Some studies in HNSCC, limited to the setting of RM disease, demonstrated a similar profile of toxicities and activity when Cetuximab was used with an EOW therapeutic schedule. In Italy, Cetuximab can be administered alone and EOW in the maintenance phase after completion of platinum-based and Cetuximab first-line treatment. ${ }^{6,8}$ Therefore, the benefits of an EOW administration would rely on the greater compliance of the patients when the treatment is in maintenance phase and extends over time.

As of today, the clinical rationale for the adoption of an EOW administration has not been investigated together with the economic consequences on the health care system. In particular, due to the scarcity of data in the literature, the budget impact (BI) of an EOW administration schedule for Cetuximab has not been assessed from the perspective of the health care service in Italy.

The objective of the present analysis is to present the clinical rationale of a different administration method of 
Cetuximab in patients diagnosed with RM HNSCC together with the economic analysis and the estimation of the potential economic benefits on the health care budget.

\section{2 | RATIONALE}

\section{1 | PK and PD data}

PK and PD for the standard weekly Cetuximab and EOW regimens were evaluated in a phase I study performed in 62 patients with metastatic colorectal cancer. ${ }^{5}$ The study was in two parts: a 6-week Cetuximab monotherapy doseescalation phase and a subsequent combination-therapy phase, during which patients received Cetuximab at the same dose/schedule as in the monotherapy phase, combined with chemotherapy. Patients in the control group received Cetuximab at a $400 \mathrm{mg} / \mathrm{m}^{2}$ initial dose, then $250 \mathrm{mg} / \mathrm{m}^{2}$ each week, whereas patients assigned to the dose-escalation group after the initial Cetuximab infusion, received 400-700 mg/ $\mathrm{m}^{2}$ EOW. The PK analysis of the different treatment groups revealed that the $700 \mathrm{mg} / \mathrm{m}^{2}$ EOW schedule deviated substantially from the other dose regimens, with higher trough concentrations in conjunction with delayed steady-state conditions, prolonged half-life, and reduced clearance. By contrast, trough concentration values for the 500 and $600 \mathrm{mg} /$ $\mathrm{m}^{2}$ EOW dosing regimen were comparable to the standard weekly regimen. Cetuximab serum concentrations and exposure increased with dose. The PK parameters terminal halflife, total plasma clearance and volume of distribution at steady state were comparable between the standard weekly and EOW 400, 500, and $600 \mathrm{mg} / \mathrm{m}^{2}$ dosing regimens. In terms of exposure, the EOW $500 \mathrm{mg} / \mathrm{m}^{2}$ dosing regimen matched more similarly the exposure of the $250 \mathrm{mg} / \mathrm{m}^{2}$ weekly schedule. Based on these data, the authors concluded that, on the whole, the closest PK match to the weekly standard regimen was provided by EOW administration of 500 or $600 \mathrm{mg} / \mathrm{m}^{2}$, with $500 \mathrm{mg} / \mathrm{m}^{2}$ being the dose of choice in terms of convenience and feasibility. Moreover, functional data derived from immunohistochemical analysis of skin biopsies added to the PK analysis and provided a biologic rationale supporting the functional equivalence of the Cetuximab weekly and EOW dosing regimens.

\section{2 | Clinical data}

The safety of the EOW regimen of Cetuximab in RM HNSCC was investigated in a phase II study performed in patients with not more than two prior cytotoxic chemotherapy regimens, randomized to receive Cetuximab EOW at $500 \mathrm{mg} / \mathrm{m}^{2}(n=35)$ or $750 \mathrm{mg} / \mathrm{m}^{2}(n=26)$ until disease progression. ${ }^{9}$ Escalating the dose to $750 \mathrm{mg} / \mathrm{m}^{2}$ did not appear to offer any obvious therapeutic advantage; therefore, this arm was prematurely closed. The administration of $500 \mathrm{mg} / \mathrm{m}^{2}$ EOW Cetuximab monotherapy was associated with grade $3 / 4$ adverse events (AEs) in $48.6 \%$ of patients. Acneiform rash was the most common grade 3 toxicity $(11 \%)$. This finding resembles the incidence of skin toxicity reported in previous studies of standard weekly dosing of Cetuximab, such as the EXTREME study, where grade 3 or higher skin reactions were reported in $9 \%{ }^{2}$ Globally, the overall response rate (ORR) was $11.4 \%$ among the $35 \mathrm{RM}$ HNSCC patients enrolled in this study and the median PFS and OS were 2.2 and 7.0 months, respectively.

In the maintenance setting, the EOW administration of Cetuximab $500 \mathrm{mg} / \mathrm{m}^{2}$ was investigated in $31 \mathrm{RM}$ HNSCC patients after chemotherapy plus weekly Cetuximab as firstline treatment. ${ }^{10}$ The safety of maintenance treatment with EOW Cetuximab was evaluated and compared with the occurrence of AEs during the previous combination therapy (chemotherapy plus weekly Cetuximab). The rate of any grade $3 / 4$ AEs was $45 \%$ and $29 \%$ in the two groups (EOW Cetuximab vs chemotherapy plus Cetuximab, respectively), whereas $16 \%$ and $19 \%$ of patients experienced grade 3/4 skin rash. In this analysis, EOW Cetuximab seemed to be well tolerated and most toxicities decreased with time during Cetuximab maintenance compared with combination therapy. No infusion reaction was observed with EOW Cetuxi$\mathrm{mab}$ at a dose of $500 \mathrm{mg} / \mathrm{m}^{2}$.

The administration of Cetuximab and Docetaxel every 2 weeks as first-line treatment of RM HNSCC was analyzed in a retrospective series of 31 patients. ${ }^{11}$ The Authors showed that grade 3/4 AEs were present in $67.7 \%$ of the patients, mainly consisting of neutropenia, hypomagnesemia, and skin rash, whereas ORR was $12.9 \%$ and median OS and PFS were 8.3 and 4 months, respectively.

The role of Cetuximab EOW as maintenance therapy was also investigated by Guigay et al. ${ }^{12}$ who have evaluated the efficacy and safety of four cycles of Docetaxel associated with Cisplatin and Cetuximab (TPEx) as first-line treatment, followed by maintenance with Cetuximab every 2 weeks in patients with RM HNSCC. Fifty-four patients were enrolled, with the most common grade 3/4 AEs being skin rash (16.6\%) and nonfebrile neutropenia $(20.4 \%)$. The primary end point was met with an ORR of $44.4 \%$; median OS and PFS were 14 and 6.2 months, respectively.

An observational French study prospectively evaluated a series of 72 patients receiving Cetuximab maintenance therapy, which was administered weekly or EOW at physician's discretion. ${ }^{13}$ Grade 3/4 skin toxicities were observed in $7.6 \%$ of the patients, whereas interestingly the 12-month PFS rate and 12-month OS rate did not differ between patients treated every 2 weeks or weekly.

Feasibility of 6 months maintenance Cetuximab after adjuvant concurrent chemoradiation plus Cetuximab in HNSCC has also been evaluated by Matuschek et al. ${ }^{14}$ Maintenance Cetuximab started after completion of chemoradiation therapy plus Cetuximab with $500 \mathrm{mg} / \mathrm{m}^{2}$ every 2 weeks over a 6-month period. Compliance to maintenance 
TABLE 1 Epidemiological parameters to identify the population diagnosed with RM HNSCC treated with Cetuximab - Italy 2017

\begin{tabular}{|llll|}
\hline Epidemiologic parameters & Model parameter (\%) & Estimated population & \multicolumn{1}{c}{ Sources } \\
\hline Residential population & & 60589445 & 22 \\
\hline HNSCC incidence & $0.015 \%$ & 9300 & 19 \\
\hline Population with RM HNSCC & $45.0 \%$ & 4185 & 20 \\
\hline $\begin{array}{l}\text { Patients treated with Cetuximab } \\
\quad \text { maintenance phase after chemotherapy }\end{array}$ & $32.3 \%$ & 1352 & 21 \\
\hline
\end{tabular}

RM HNSCC, recurrent and/or metastatic head and neck squamous cell cancer.

Cetuximab was quite satisfactory: $80 \%$ were still on Cetuximab after 3 months and 63\% after 5 months; $48 \%$ completed 6 months maintenance therapy.

\section{I ECONOMIC CONSEQUENCES}

An excel-based model was developed to estimate the potential economic benefits of EOW compared to weekly administration of Cetuximab among patients with RM HNSCC. A review of the epidemiological and economic literature was conducted to identify relevant information to include in the analysis. The model was then implemented following the guidelines suggested by the International Society of Pharmacoeconomics and Outcomes Research (ISPOR). ${ }^{15,16}$

\section{1 | Comparison scenarios}

In coherence with the current administration schedule, a base case scenario was set considering $100 \%$ of patients being treated with a weekly administration of Cetuximab. ${ }^{17}$ In the comparison scenario, the base case administration was replaced with EOW administration at $50 \%$ or $100 \%$ for the maintenance therapy only. Specifically, in the base case scenario, the schedule included a dose of $250 \mathrm{mg} /$ body surface area (BSA) ${ }^{17}$ compared with one dose of $500 \mathrm{mg} / \mathrm{BSA}$ every 2 weeks (alternative scenario).

Moreover, the expense simulations have been broken down in cost analyses per milligram of drug used (base case) and per required ampoule (sensitivity analysis). The model assumed an average BSA of $1.8 \mathrm{~m}^{2.18}$ Details on the treatment schedules are reported in Table 2 .

\section{2 | Epidemiological parameters}

As a first step, the size of the eligible population was identified from the national perspective according to the therapeutic indication in Italy. As reported in Table 1, incidence rates provided by the Italian Association of Cancer Registries ${ }^{19}$ were used to estimate the cohort of individuals annually diagnosed with HNSCC cancer. Moreover, it was estimated that about $45 \%$ of these patients were diagnosed with RM disease. $^{20}$

Being the model focused on patients treated with Cetuximab alone in the maintenance setting, we assumed that about
$32 \%$ of patients were treated with first line platinum-based chemotherapy plus Cetuximab ${ }^{21}$ and that the portion undergoing Cetuximab monotherapy in maintenance phase was $45 \% .^{2}$ As a result, the model estimated a cohort of patients treated with Cetuximab equivalent to 609 patients a year (Table 1). ${ }^{2}$

The second step concerned the definition of the time horizon to consider in the analysis. Because of the limited PFS of RM HNSCC, we set a 2- to 4-month therapy time horizon.

\section{3 | Cost parameters}

With reference to the estimation of drug cost, the price of Cetuximab has been used net of discounts by law and according to the dosing of the two treatment schedules (Table 2). The model considered both the cost $/ \mathrm{mg}$ and the cost/ampoule, according to the treatment schedules and the ampoules required for an average BSA of $1.8 \mathrm{~m}^{2}$.

Furthermore, the cost associated with patients' management was estimated. According to this approach, the cost of medical examinations required for drug administration and patient management was included in addition to the indirect costs associated with the loss of productivity (absence from work) of the patient or caregiver.

Specifically, the model assumed a cost of $€ 85 /$ medical examination for each administration, including the cost of the physician, nurse, consumption material, for the drug administration, and distribution by the hospital pharmacy. ${ }^{23}$

TABLE 2 Parameters of patient definition and therapy cost

\begin{tabular}{|lll|}
\hline $\begin{array}{l}\text { Parameters of patient definition } \\
\text { H\&N R/M }\end{array}$ & Parameter & Source \\
\hline BSA patient, ${ }^{2}$ & 1.8 & 18 \\
\hline Weekly ampoule CET_weekly/250 mg/BSA & $5.0 / 450 \mathrm{mg}$ & 17 \\
\hline Weekly ampoule CET_EOW/500 mg/BSA & $9.0 / 900 \mathrm{mg}$ & Assumption \\
\hline $\begin{array}{l}\text { Number of lost working days/medical } \\
\quad \text { examination }\end{array}$ & 1.0 & Assumption \\
\hline $\begin{array}{l}\text { Cost parameters } \\
\text { Ampoule price } 100 \mathrm{mg}\end{array}$ & Cost & Source \\
\hline $\begin{array}{l}\text { Cost of medical examination/ } \\
\text { administration }\end{array}$ & $€ 153.6$ & AIFA \\
\hline \begin{tabular}{l} 
Cost of working day Italy \\
\hline
\end{tabular} & $€ 85.0$ & 23 \\
\hline
\end{tabular}

BSA, body surface area; CET, Cetuximab; EOW, every other week. 
TABLE 3 Budget impact results, base case vs EOW 50\% scenario,

2 months

\begin{tabular}{|c|c|c|c|}
\hline \multirow[b]{2}{*}{ Cost items } & \multicolumn{3}{|l|}{ Expense } \\
\hline & Base case $(€)$ & $\begin{array}{c}\text { EOW } 50 \% \\
\text { scenario }(€)\end{array}$ & $\begin{array}{l}\text { Budget } \\
\text { impact }(€)\end{array}$ \\
\hline $\begin{array}{l}\text { Drug cost } \\
\quad \text { (calculation/mg) }\end{array}$ & $€ 2990322$ & $€ 2990322$ & $€ 0$ \\
\hline $\begin{array}{l}\text { Management cost/ } \\
\text { administration }\end{array}$ & $€ 413808$ & $€ 310356$ & $-€ 103452$ \\
\hline Indirect costs & $€ 974445$ & $€ 730833$ & $-€ 243611$ \\
\hline \multirow[t]{2}{*}{ Total expense } & $€ 4378574$ & $€ 4031511$ & $-€ 347063$ \\
\hline & 1 week & $\begin{array}{r}\text { EOW } 50 \% \\
\text { scenario }\end{array}$ & $\begin{array}{l}\text { Tot. no. of } \\
\text { treated patients }\end{array}$ \\
\hline $\begin{array}{l}\text { Base case treated } \\
\text { patients }\end{array}$ & 609 & 0 & 609 \\
\hline $\begin{array}{l}50 \% \text { treated patients } \\
\text { scenario }\end{array}$ & 304 & 304 & 609 \\
\hline
\end{tabular}

With reference to the indirect costs, the model assumed the loss of a working day every time the drug is administered to the patient in the hospital setting. Such assumption was based on the hypothesis that, when drug is administered, the whole working day is lost either by the patient or the caregiver. Calculations were made considering an average salary/hour of $€ 27.8^{24}$ that corresponded to a daily salary of $€ 200.2^{24,25}$ before tax (Table 2).

Finally, the model does not consider efficacy and safety differences between the two schedules.

\subsection{Sensitivity analysis}

Deterministic one-way sensitivity analysis was performed to model the uncertainty of the parameters and the consequent variability of the results. Following this approach, the results of the BI model have been obtained by varying one parameter of the model at once, depending on the variability observed in the literature or assumed by the authors. Specifically, the following scenarios have been considered:

- RM-HNSCC patients with maintenance treatment with Cetuximab $\quad$ base case $=45.0 \%$ ): $\operatorname{Min}=35 \%-\operatorname{Max}=$ $55 \%$

- Cost of estimated drug by number of ampoules required for the administration (base case $=$ cost $/ \mathrm{mg}$ )
- Working days lost/visit (base case $=1$ day lost): Min $=0-$ Max $=2$

\section{4 | RESULTS}

The model estimated a total of 609 patients diagnosed with RM-HNSCC and subjected to treatment with Cetuximab once a week in the base case scenario. In the next paragraphs, the comparison between the base case and an EOW administration schedule applied to $50 \%$ and $100 \%$ of the eligible population is illustrated.

\section{1 | Base case vs EOW 50\% scenario}

Table 3 illustrates the results in terms of BI after 2 months, considering 609 weekly treated patients vs 304 weekly patients and 304 bi-monthly treated patients. Considering an average BSA of $1.8 \mathrm{~m}^{2}$, the two strategies (weekly administration of $250 \mathrm{mg} / \mathrm{BSA}$ vs EOW $500 \mathrm{mg} / \mathrm{BSA}$ ) did not differ in terms of the cost of the drug/month. The model estimated a cost reduction of $€ 347000$, of which over $70 \%$ was attributable to indirect costs. Administration costs showed a smaller saving of approximately $€ 243000$ (Figure 1). In addition, the model estimated that, after 4 months of treatment, the cost reduction would increase to $€ 694000$.

\subsection{Base case vs EOW $100 \%$ scenario}

In this scenario, the BI of EOW strategy was compared with a weekly strategy for patients with RM-HNSCC at 2 and 4 months considering same drug expense for the two treatment strategies. As a result, the model estimated a cost reduction of $€ 694000$, of which about $70 \%$ is due to indirect costs after 2 months (Table 4; Figure 2). After 4 months of treatment, the cost reduction would increase to $€ 138$ million considering the cost/mg and to $€ 213$ million considering the cost/vial of drug.

\section{3 | Sensitivity analysis results for RM-HNSCC patients}

Figure 3 illustrates the variables with the highest impact on the model results. One-way sensitivity analysis showed as

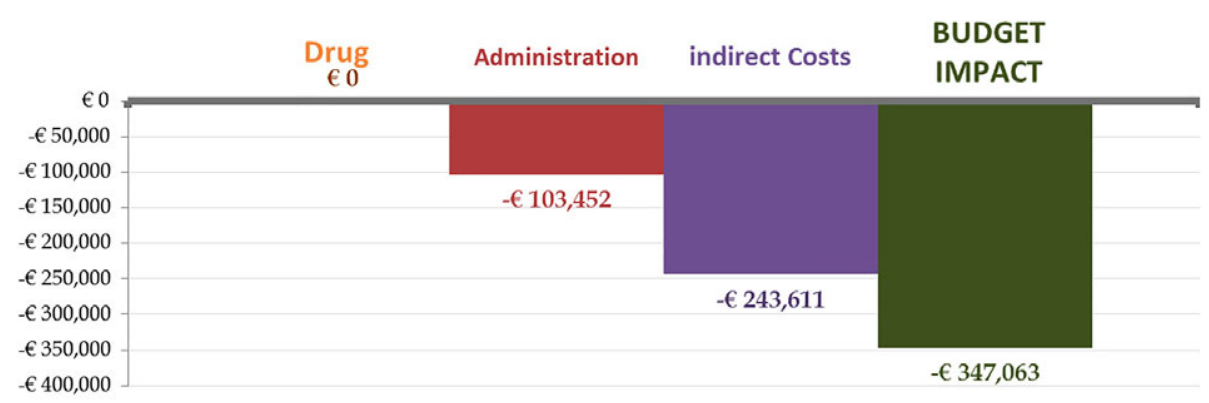

FIGURE 1 Budget impact results, base-case vs EOW 50\% scenario, per cost item (€). RM HNSCC, recurrent and/or metastatic head and neck squamous cell cancer; EOW, every other week [Color figure can be viewed at wileyonlinelibrary.com] 
TABLE 4 Budget impact results, base case vs EOW 100\% scenario, 2 months

\begin{tabular}{|c|c|c|c|}
\hline \multirow{2}{*}{ Cost items } & \multicolumn{3}{|l|}{ Expense } \\
\hline & $\overline{\text { Base case }(€)}$ & EOW $100 \%$ scenario $(€)$ & Budget impact $(€)$ \\
\hline Drug cost (calculation/mg) & $€ 2990322$ & $€ 2990322$ & $€ 0$ \\
\hline Management cost/administration & $€ 413808$ & $€ 206904$ & $-€ 206904$ \\
\hline Indirect costs & $€ 974445$ & $€ 487222$ & $-€ 487222$ \\
\hline \multirow[t]{2}{*}{ Total expense } & $€ 4378574$ & $€ 3684448$ & $-€ 694126$ \\
\hline & 1 week & EOW $100 \%$ scenario & Tot. No. of treated patients \\
\hline Base case treated patients & 609 & 0 & 609 \\
\hline $100 \%$ treated patients scenario & 0 & 609 & 609 \\
\hline
\end{tabular}

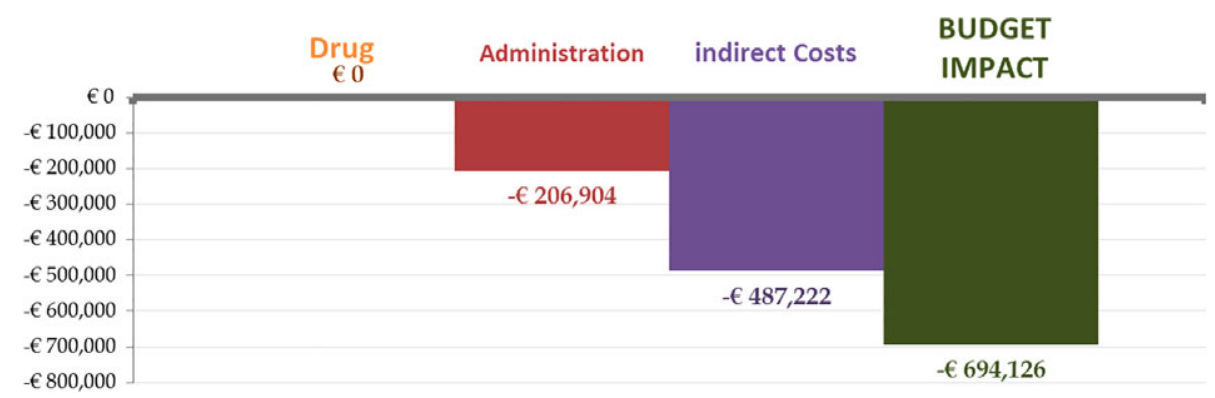

FIGURE 2 Budget impact results, base-case vs EOW 100\% scenario, per cost item (€). RM HNSCC, recurrent and/or metastatic head and neck squamous cell cancer; EOW, every other week [Color figure can be viewed at wileyonlinelibrary.com]

the model was most sensitive to the number of working days lost by patient or caregivers and when the drug cost was per vial instead of milligram. (Figure 3).

\section{5 | DISCUSSION}

In this analysis, we focused on the economic benefit of an EOW administration of Cetuximab vs a weekly schedule, as maintenance therapy.
Patients with RM-HNSCC not amenable to surgical salvage or radiation therapy may receive a combination of platinum-based chemotherapy and Cetuximab for a maximum of 6 cycles, followed by maintenance Cetuximab in case of clinical benefit and good tolerability. The weekly schedule of Cetuximab was used in the pivotal Extreme trial and thereby adopted in the clinical practice.

As confirmed by the PK data, the EOW $500 \mathrm{mg} / \mathrm{m}^{2}$ schedule was similar in terms of exposure to the $250 \mathrm{mg} / \mathrm{m}^{2}$ weekly dose; clinical data have confirmed the feasibility and

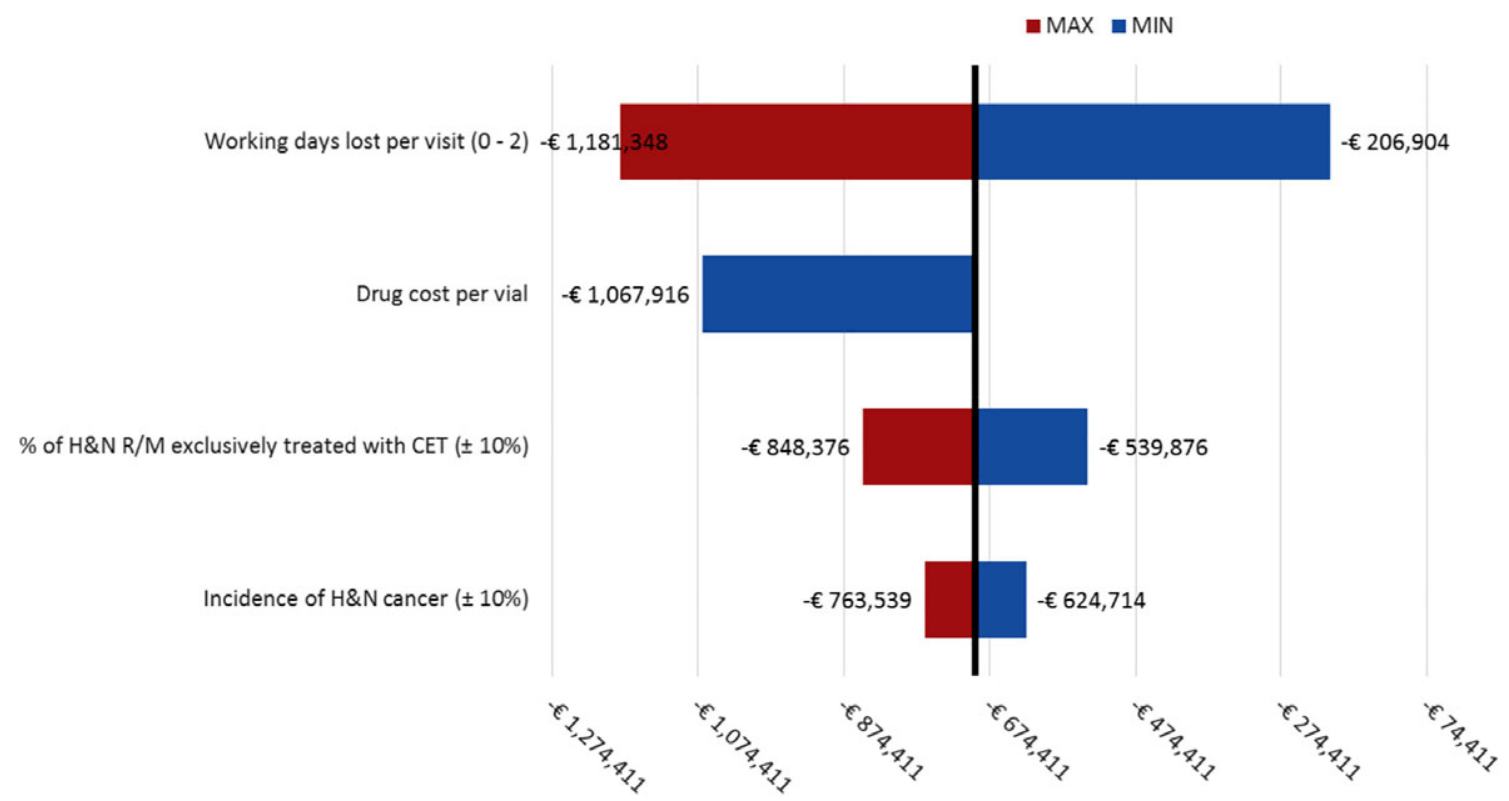

FIGURE 3 One-way sensitivity analysis—case base budget impact vs EOW 100\% scenario. H\&N, head and neck; RM HNSCC, recurrent and/or metastatic head and neck squamous cell cancer; EOW, every-other week [Color figure can be viewed at wileyonlinelibrary.com] 
activity of this approach, so justifying its broader use. The possible benefits of an EOW schedule in the maintenance phase rely on a better compliance of the patients, subject to a high number of cycles in the previous months; moreover, this advantage could reflect also into a benefit in the quality of life of the patients, receiving endovenous administration phased in longer time.

In our analysis, we showed that this simplified schedule could also reduce the costs of treatments both for the health system (direct costs) and for the society (indirect costs). The latter accounted for the major part of the potential savings following the adoption of an EOW administration of Cetuximab in Italy. This result was obtained assuming that after chemotherapy and Cetuximab, patients would benefit from an improvement of symptoms in the maintenance phase that would allow to gradually resume working.

Therefore, considering the preclinical and clinical premises and the economic benefits, we would suggest that the EOW schedule should be adopted as possible schedule of administration of Cetuximab in the maintenance phase, assuming that the disease remains controlled and the AEs are well tolerated.

Some limitations have to be underlined in our analysis. First, the epidemiological parameters were based on the data published in national reports, therefore with reference to the considered diseases; the number of treated patients with Cetuximab in Italy could be either overestimated or underestimated. However, in sensitivity analyses, these variables have been changed in a representative range of the national reality, allowing interval estimates able to represent a minimum and maximum impact of plausible expense.

Second, the model may have underestimated the real cost of patients treated with RM-HNSCC as it took into account a limited number of cost items. In particular, the model considered only the cost of Cetuximab and its administration and indirect costs deriving from patient or caregiver's absence from work. Transportation costs were not included due to the absence of a specific transportation program dedicated to chemotherapy administration patients funded by NHS. Also the costs due to AEs and/or disease progression and presenteeism or absenteeism for AEs were not included. However, in this case, we assumed that they would be identical between the two comparisons as confirmed by the therapeutic equivalence both in terms of safety and efficacy of the two administration methods of Cetuximab and hence not affecting the economic estimation. Finally, we did not include other possible economic saving in the EOW schedule, such as the possibility to reduce the drug waste in case of the use of the drug contained in vial of fixed dosage, which cannot be completely finished.

Finally, a third limitation is the lack of information on the lost working days in the two groups of treatment. The model conservatively assumed that the patients or caregivers lost at least one working day. However data may be underestimated due to the risk of AEs causing absence or reduced productivity or even a permanent exit from the labor market. In addition, caregivers could be also involved for an extra day after the day of the treatment.

In conclusion, the model represents a first attempt to quantify the economic impact of a change in treatment schedules of Cetuximab in Italy. Following the clinical rationale for its adoption, the analysis assessed the potential impact of an EOW administration from a societal perspective in which indirect costs resulted as the main driver. However, the new treatment strategy would also free resources in terms of lower hospital admissions that may be efficiently reallocated to maximize the work/hours of hospital staff and therefore have a positive impact from the hospital perspective.

\section{ORCID}

\section{P Bossi (D) https://orcid.org/0000-0003-0135-0224}

\section{REFERENCES}

1. Bonner JA, Harari PM, Giralt J, et al. Radiotherapy plus cetuximab for squamous-cell carcinoma of the head and neck. $N$ Engl J Med. 2006;354: 567-578.

2. Vermorken JB, Mesia R, Rivera F, et al. Platinum-based chemotherapy plus cetuximab in head and neck cancer. $N$ Engl J Med. 2008;359:1116-1127.

3. Markovic A, Chung $\mathrm{CH}$. Current role of EGF receptor monoclonal antibodies and tyrosine kinase inhibitors in the management of head and neck squamous cell carcinoma. Expert Rev Anticancer Ther. 2012;12:1149-1159.

4. Tabernero J, Ciardiello F, Rivera F, et al. Cetuximab administered once every second week to patients with metastatic colorectal cancer: a two-part pharmacokinetic/pharmacodynamic phase I dose-escalation study. Ann Oncol. 2010;21:1537-1545.

5. Tabernero J, Pfeiffer P, Cervantes A. Administration of cetuximab every 2 weeks in the treatment of metastatic colorectal cancer: an effective, more convenient alternative to weekly administration? Oncologist. 2008;13: 113-119.

6. Pfeiffer P, Nielsen D, Bjerregaard J, Qvortrup C, Yilmaz M, Jensen B. Biweekly cetuximab and irinotecan as third-line therapy in patients with advanced colorectal cancer after failure to irinotecan, oxaliplatin and 5-fluorouracil. Ann Oncol. 2008;19:1141-1145.

7. Mrabti H, De la Fouchardiere C, Desseigne F, et al. Irinotecan associated with cetuximab given every 2 weeks versus cetuximab weekly in metastatic colorectal cancer. J Cancer Res Ther. 2009;5:272-276.

8. Fontes M. The process of transformation of scientific and technological knowledge into economic value conducted by biotechnology spin-offs. Technovation. 2005;25:339-347.

9. Fury MG, Sherman E, Lisa D, et al. A randomized phase II study of cetuximab every 2 weeks at either 500 or $750 \mathrm{mg} / \mathrm{m}^{2}$ for patients with recurrent or metastatic head and neck squamous cell cancer. J Natl Compr Canc Netw. 2012;10:1391-1398.

10. Bossi P, Kornek G, Lanzetta G, et al. Safety and feasibility of every-otherweek maintenance cetuximab after first-line chemotherapy in patients with recurrent or metastatic head and neck squamous cell cancer. Head Neck. 2013;35:1471-1474.

11. Posch D, Fuchs H, Kornek G, et al. Docetaxel plus cetuximab biweekly is an active regimen for the first-line treatment of patients with recurrent/metastatic head and neck cancer. Sci Rep. 2016;6:32946.

12. Guigay J, Fayette J, Dillies AF, et al. Cetuximab, docetaxel, and cisplatin as first-line treatment in patients with recurrent or metastatic head and neck squamous cell carcinoma: a multicenter, phase II GORTEC study. Ann Oncol. 2015;26:1941-1947.

13. Guigay J, Chamorey E, Céruse $P$, et al. Observational study of the cetuximab relative dose intensity (RDI) in the first-line treatment of recurrent and/or metastatic squamous cell carcinoma of the head and neck (R/M SCCHN): 
data on the maintenance and every two weeks use (DIRECT study). Ann Oncol. 2016;27(6):328-350. https://doi.org/10.1093/annonc/mdw376.

14. Matuschek C, Bolke E, Belka $\mathrm{C}$, et al. Feasibility of 6-month maintenance cetuximab after adjuvant concurrent chemoradiation plus cetuximab in squamous cell carcinoma of the head and neck. Strahlenther Onkol. 2013;189: 625-631.

15. Mauskopf JA, Sullivan SD, Annemans L, et al. Principles of good practice for budget impact analysis: report of the ISPOR Task Force on good research practices--budget impact analysis. Value Health. 2007;10:336-347.

16. Sullivan SD, Mauskopf JA, Augustovski F, et al. Budget impact analysisprinciples of good practice: report of the ISPOR 2012 Budget Impact Analysis Good Practice II Task Force. Value Health. 2014;17:5-14.

17. European Medicine Agency (EMA). Information document of Cetuximab. In http://www.ema.europa.eu/ema/index.jsp?curl=pages/medicines/human/ medicines/000558/human_med_000769.jsp\&mid=WC0b01ac058001d 124 (ed). 2004

18. Pinto C, Di Fabio F, Rosati G, et al. Observational study on quality of life, safety, and effectiveness of first-line cetuximab plus chemotherapy in KRAS wild-type metastatic colorectal cancer patients: the ObservEr Study. Cancer Med. 2016;5:3272-3281.

19. Tumori AIdR. I numeri del cancro in Italia. 2016.

20. Sacco AG, Cohen EE. Current treatment options for recurrent or metastatic head and neck squamous cell carcinoma. J Clin Oncol. 2015;33:3305-3313.

21. IMS. DataView Multi Channel MO, Vendite Volume e Valore, Sell-in Cal. Totale Italia, Tutti i Canali Aprile 2013-Ottobre. 2016.
22. Istituto Nazionale di Statistica (ISTAT). Popolazione residente al 1 Gennaio 2017. In Statistiche Demografiche. 2017.

23. Mennini FS, et al. Terapia orale: Analisi di minimizzazione dei costi presso una struttura ospedaliera della Regione Laziovs: Analisi di minimizzazione dei costi presso una struttura ospedaliera della Regione Lazio terapia infusionale nel trattamento del cancro del colon retto localmente avanzato o avanzato: Analisi di minimizzazione dei costi presso una struttura ospedaliera della Regione Lazio. Pharmacoeconomics-Italian Research Articles. 2012; 14(2):121-129.

24. Eurostat. Labour cost levels by NACE Rev. 2 activity. 2017.

25. Istat. http://dati.istat.it/Index.aspx ?DataSetCode=DCCV_ORELAVMED. 2016.

How to cite this article: Mennini $F$, Marcellusi A, Fabiano G, Platini F, Bossi P. Rationale and budget impact of bimonthly use of Cetuximab in patients with recurrent and/or metastatic head and neck cancer. Head \& Neck. 2019;1-7. https://doi.org/10.1002/hed. 25481 